\title{
3d synthetic aperture radar image
}

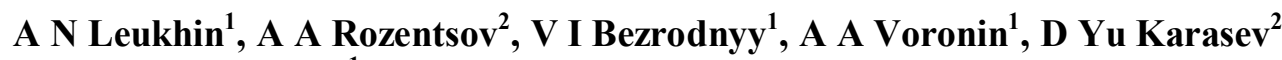 \\ and $\mathbf{N} A$ Kokovihina ${ }^{1}$ \\ ${ }^{1}$ Mari State University, Lenin square 1, Yoskar-Ola, Russia, 424000 \\ ${ }^{2}$ Volga State University of Technology, Lenin square 3, Yoskar-Ola, Russia, 424000
}

\begin{abstract}
Synthetic aperture radar (SAR) is a coherent active microwave imaging method. In remote sensing it is used for mapping the scattering properties of the Earth's surface in the respective wavelength domain. The algorithms for the formation of 3D radar images in multiposition interferometric systems for remote sensing of the Earth are considered. Examples of reconstruction of the relief map for systems with one and two transmit antenna are presented.
\end{abstract}

\section{Introduction}

The application of interferometric data processing to obtain information about the terrain and its changes, implementation of high resolution $(1-3 \mathrm{~m})$ regimes have become the main trends in the development of modern radar systems for space observation. Such processing of space-based synthetic aperture radar`s data includes the following steps: synthesis a pair of complex radar images of the same surface region, their spatial overlap with the formation of an interferogram; phase noise filtering on the obtained interferogram; deployment of the phase of the interferogram and its full geocoding (recalculation of the values of the expanded phase in the values of the relief heights and the transition from the flight coordinate system to any cartographic projection).

The purpose of this paper is to review the methods of interferometric data processing to obtain $3 \mathrm{~d}$ synthetic radar image of a surface model.

It is necessary to first describe the basics of $3 \mathrm{~d}$ SAR imaging and explain its features.

A second antenna is installed on an aircraft at a certain distance from the first antenna in order to enable forming a three-dimensional map of the underlying surface mode in synthetic aperture radar. As a rule, the spacing can be carried out either in height or in a horizontal plane perpendicular to the direction of flight.

There are several options for the operation of SAR in 3D mode [1]:

1. Radiation is performed through both antennas by turns.

2. Radiation is made through one antenna (for example, $A_{1}$ ), and receiving is performed through two antennas $A_{1}$ and $A_{2}$.

3. On the radiation, the transmitter operates on one of its own antennas $\left(A_{3}\right)$, the receivers have two own antennas $A_{1}$ and $A_{2}$.

$3 \mathrm{D}$ images of surface are analysed by hypertrace transformation [2].

Let's consider the basic geometrical relations necessary for calculation of objects' height in SAR 3D mode. 


\section{2. "Two transmitters, two receivers" system}

Figure 1 shows the basic geometric equations for the 3D SAR system, for the case when the aircraft is equipped with two transmitter and two receivers, operating through its own spaced from each other antenna.

Using the basis of the Pythagorean theorem we can write:

$$
\left\{\begin{array}{l}
\left(H_{1}-h\right)^{2}+r_{1}^{2}=R_{1}^{2} \\
\left(H_{2}-h\right)^{2}+\left(r_{1}+d\right)^{2}=R_{2}^{2}
\end{array}\right.
$$

Values of the heights $H_{1}$ and $H_{2}$ of the antennas and antenna spacing $d$ in the horizontal plane can be considered known while SAR is working. Also known and sloped range $R_{1}$ and $R_{2}$. You need to find the horizontal distance $r_{1}$ and the height of the object $h$. Before solving the equation (1) we can first calculate $r_{1}$ and then determine $h$ or vice versa. The solution to the system is determined by the ratio between the values $d$ and $\left|H_{1}-H_{2}\right|$. If $\left|H_{1}-H_{2}\right|>d$ you choose the first method, otherwise the second. Two solutions are obtained when you use each method. A preference in favor of a solution that gives value $r_{1}$ least different from the values of the horizontal range, designed for zero height $r_{1}^{(0)}=\left(R_{1}^{2}-H_{1}^{2}\right)^{1 / 2}$, i.e. $\left|r_{1}-r_{1}^{(0)}\right| \rightarrow \min [3]$.

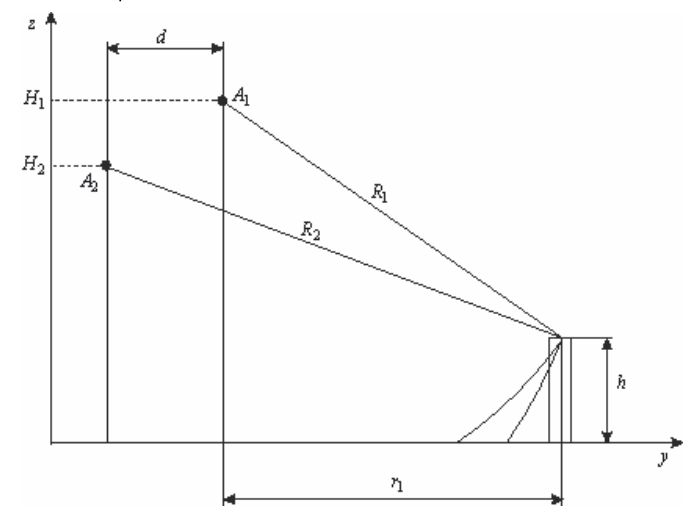

Figure 1. Basic geometric equations in "two transmitters, two receivers" system.

Possible solutions of the equation (1) are defined by equations (2) and (3):

$$
\begin{gathered}
r 1=-\left(H 1^{2} \cdot d+H 2^{2} \cdot d+R 1^{2} \cdot d-R 2^{2} \cdot d+d^{3}+2 \cdot H 1 \cdot H 2 \cdot d+\right. \\
+\left(H 1^{2}-2 \cdot H 1 \cdot H 2+H 2^{2}-R 1^{2}+2 \cdot R 1 \cdot R 2-R 2^{2}+d^{2}\right)^{1 / 2} . \\
\cdot\left(2 \cdot H 1 \cdot H 2-H 1^{2}-H 2^{2}+R 1^{2}+2 \cdot R 1 \cdot R 2+R 2^{2}-d^{2}\right)^{1 / 2} . \\
\cdot(H 2-H 1)) \cdot \frac{1}{2 \cdot\left(H 1^{2}-2 \cdot H 1 \cdot H 2+H 2^{2}+d^{2}\right)} ; \\
h=-\frac{H 2^{2}-H 1^{2}+R 1^{2}-R 2^{2}+d^{2}+2 \cdot r 1 \cdot d}{2 \cdot H 1-2 \cdot H 2} \\
h=-\left(H 1 \cdot d^{2}+H 2 \cdot d^{2}+H 1^{3}+H 2^{3}-H 1 \cdot H 2^{2}-H 1^{2} \cdot H 2-\right. \\
-H 1 \cdot R 1^{2}+H 1 \cdot R 2^{2}+H 2 \cdot R 1^{2}-H 2 \cdot R 2^{2}+\cdot d \cdot \\
\cdot\left(H 1^{2}-2 \cdot H 1 \cdot H 2+H 2^{2}-R 1^{2}+2 \cdot R 1 \cdot R 2-R 2^{2}+d^{2}\right)^{1 / 2} . \\
\left.\quad\left(2 \cdot H 1 \cdot H 2-H 1^{2}-H 2^{2}+R 1^{2}+2 \cdot R 1 \cdot R 2+R 2^{2}-d^{2}\right)^{1 / 2}\right) . \\
\quad \frac{1}{2 \cdot\left(H 1^{2}-2 \cdot H 1 \cdot H 2+H 2^{2}+d^{2}\right)} ; \\
r=-\frac{R 1^{2}-R 2^{2}+d^{2}+(H 2-h)^{2}-(H 1-h)^{2}}{2 \cdot d}
\end{gathered}
$$


On the practice ranges $R_{1}$ and $R_{2}$ are known with an item resolution accuracy, so the calculations of value $h$ and $r_{1}$ use value $\Delta R=R_{2}-R_{1}$, calculated on the basis of the measurement result of the phase difference

$$
\Delta R=\frac{\Delta \varphi^{(w)}}{4 \pi} \lambda=\frac{\varphi_{2}-\varphi_{1}}{4 \pi} \lambda
$$

where $\varphi_{1}$ and $\varphi_{2}$ - phases of the signals received from the first and second antennas, respectively; $\lambda$ wave`s length.

Because the value of the phase shift is in the range $[0,2 \pi)$, as a rule, there is ambiguity of the measurement values $\Delta R$. To fix it uses the "unwrap" phase. Consider possible approaches to its implementation [4].

The first approach is based on the preliminary construction of the dependence of phase shifts to the zero level, depending on slant range to the antenna $A_{1}[5]$ :

$$
\Delta \varphi^{(0)}\left(R_{1}\right)=4 \pi \Delta R^{(0)}\left(R_{1}\right) / \lambda=4 \pi\left(\left(H_{2}^{2}+\left(\left(R_{1}^{2}-H_{1}^{2}\right)^{1 / 2}+d\right)^{2}\right)^{1 / 2}-R_{1}\right) / \lambda
$$

Then the value of the phase shift $\Delta \varphi^{(u)}\left(R_{1}\right)$ used to calculate the difference between the sloping distances, determined from the relationship:

$$
\Delta \varphi^{(u)}\left(R_{1}\right)=\Delta \varphi^{(w)}+\operatorname{trunc}\left(\frac{\Delta \varphi^{(0)}\left(R_{1}\right)}{2 \pi}\right) 2 \pi
$$

The value of the slant range $R_{2}$ used for the calculation $h$ and $r_{1}$ is determined from the relation:

$$
R_{2}=R_{1}+\frac{\Delta \varphi^{(u)}}{4 \pi} \lambda \pm 2 \pi m
$$

To determine the value $m$, you can use the following approach. Typically, the height difference between adjacent pixels is relatively small and we can assume that the height of the object in some neighborhood is constant. Presented according to $m$ estimates of the altitude differences in the neighboring pixels have a pronounced minimum, and calculations show that this minimum is achieved when the value of the parameter $\hat{m}$ corresponding to the true values of the altitude and slant range. The algorithm of the calculation value $\hat{m}$ is the following:

1. Around the current image point with coordinates $x_{0}, y_{0}$, set the gate;

2. Sets the range of values $m: m=m_{\min } . . m_{\max }$

3. For each point in the gate with coordinates $x, y$, a values $r_{x, y}$ and $h_{x, y}$ are calculated with current value $m$;

4. Calculate total measurement error of the heights and horizontal distances. Terms $\left(y_{1}-y_{0}\right)$ and $\left(y_{2}-y_{0}\right)$ take into account the current offset of pixels in the horizontal range:

$$
\begin{aligned}
& \Delta h_{m}=\sum_{x_{1}=x_{0}-d x}^{x_{0}+d x} \sum_{y_{1}=y_{0}-d y}^{y_{0}+d y} \sum_{x_{2}=x_{0}-d x}^{x_{0}+d x} \sum_{y_{2}=y_{0}-d y}^{y_{0}+d y}\left|h_{x_{1}, y_{1}}-h_{x_{2}, y_{2}}\right| \\
& \Delta r_{m}=\sum_{x_{1}=x_{0}-d x}^{x_{0}+d x} \sum_{y_{1}=y_{0}-d y}^{y_{0}+d y} \sum_{x_{2}=x_{0}-d x}^{x_{0}+d x} \sum_{y_{2}=y_{0}-d y}^{y_{0}+d y} \Delta y_{x 1, x 2, y_{1}, y_{2}} \\
& \Delta y_{x 1, x 2, y 1, y 2}=\left|\left(r_{x_{1}, y_{1}}-\left(y_{1}-y_{0}\right)\right)-\left(r_{x_{2}, y_{2}}-\left(y_{2}-y_{0}\right)\right)\right|
\end{aligned}
$$

5. As a result, selects the value $\hat{m}$ at which

$$
\Delta h_{\hat{m}} \rightarrow \min , \Delta \kappa_{\hat{m}} \rightarrow \min
$$

Because of spacing antennas, the resulting images have some mutual shift, and its magnitude will depend on the range. In this regard, before calculating the elevation, you must perform the mutual correction of the shifts of image elements. For this calculate values of distances $R_{2}$ corresponding to 
the range $R_{1}$ on the zero level and overwrite the elements of the second array with the ranges $R_{2}$ in cells that correspond to values in the range $R_{1}$ [6]:

$$
\begin{gathered}
J_{R_{1}, x}^{(\kappa o p)}=J_{R_{2}, x}^{(2)} \\
R_{2}=\left(H_{2}{ }^{2}+\left(\left(R_{1}^{2}-H_{1}^{2}\right)^{1 / 2}+d\right)^{2}\right)^{1 / 2}
\end{gathered}
$$

where $J_{R_{2}, x}^{(2)}$ - image from the second antenna.

Figure 2 shows an example of the recovery bump maps for the considered case when the following system parameters: $H_{1}=H_{2}=10 \mathrm{~m}, d=5 \mathrm{~m}$ the maximum altitude of the relief $5 \mathrm{~m}$.

(a)

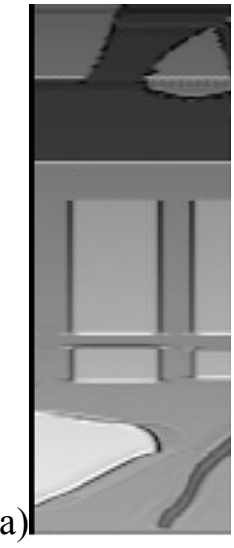

(b)

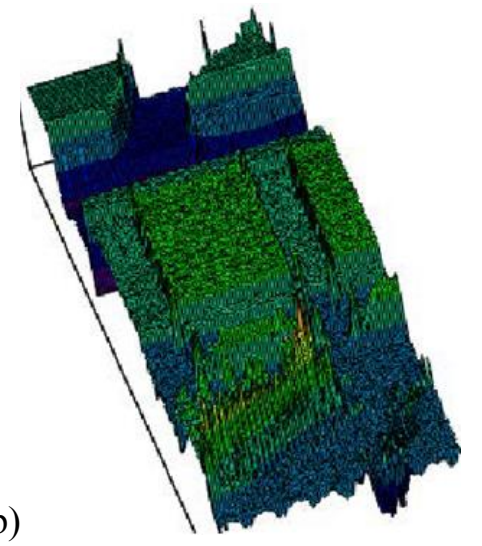

Figure 2. An example of the recovery of the terrain surface. $a$ - synthesized image, $b-3 D$ relief of the surface.

\section{3. "Two transmitters, one receiver" system}

Figure 3 shows the basic geometric equations for the 3D SAR system, for the case when an aircraft equipped with one transmitter operating, for example, via an antenna $A_{1}$, and two receivers, operating through its own separated antennas $A_{1}$ and $A_{2}$.

In this system a signal between antennas $A_{1}$ and $A_{2}$ the point on the object surface takes place in two ways $S_{1}=2 R_{1}$ and $S_{2}=R_{1}+R_{2}$ respectively. In the simulation of the hologram samples of the signal in the first image is recorded with a pixel corresponding to the distance $R_{1}$, and a second hologram pixel $\frac{R_{1}+R_{2}}{2}$.

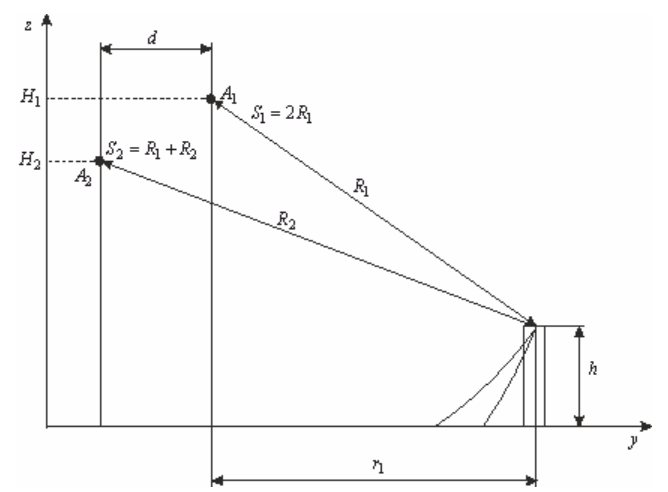

Figure 3. Basic geometric equations in "two transmitters, one receiver" system.

The value of the path $S_{2}$ is determined based on the known path $S_{1}$ and phase difference due to antenna spacing: 


$$
S_{2}=S_{1}+\frac{\Delta \varphi}{2 \pi} \lambda m, m=\ldots,-2,-1,0,1,2, \ldots
$$

Knowing the values of the parameters $S_{1}, S_{2}, H_{1}, H_{2}, d$, you can find value and a horizontal range $r_{1}$ :

$$
\begin{gathered}
D 2=\left(-d^{2} \cdot\left(-d^{2} \cdot S 1^{2}-2 \cdot H 1^{2} \cdot S 2^{2}-2 \cdot H 2^{2} \cdot S 2^{2}+2 \cdot S 1 \cdot d^{2} \cdot S 2+S 2^{4}-H 2^{2} \cdot S 1^{2}-\right.\right. \\
-4 \cdot H 1^{3} \cdot H 2-2 \cdot d^{2} \cdot S 2^{2}-H 1^{2} \cdot S 1^{2}+d^{4}+2 \cdot H 2^{2} \cdot d^{2}+H 1^{4}+H 2^{4}+2 \cdot H 1^{2} \cdot d^{2}+ \\
+6 \cdot H 1^{2} \cdot H 2^{2}+2 \cdot S 1 \cdot S 2 \cdot H 1^{2}-2 \cdot S 1 \cdot S 2^{3}-4 \cdot H 1 \cdot H 2 \cdot d^{2}-4 \cdot H 1 \cdot H 2^{3}+4 \cdot H 1 \cdot H 2 \cdot S 1^{2}- \\
\left.\left.-4 \cdot H 1 \cdot H 2 \cdot S 1 \cdot S 2+2 \cdot H 2^{2} \cdot S 1 \cdot S 2+4 \cdot H 1 \cdot H 2 \cdot S 2^{2}+S 1^{2} \cdot S 2^{2}\right)\right)^{0.5} \\
h=\frac{-H 1 \cdot S 1 \cdot S 2+H 2 \cdot d^{2}-H 1^{2} \cdot H 2+H 1^{3}+H 2^{3}+H 1 \cdot d^{2}-H 2 \cdot S 2^{2}}{2 \cdot\left(H 1^{2}+H 2^{2}+d^{2}-2 \cdot H 1 \cdot H 2\right)}+ \\
+\frac{H 1 \cdot S 2^{2}+H 2 \cdot S 1 \cdot S 2-H 1 \cdot H 2^{2}-D 2}{2 \cdot\left(H 1^{2}+H 2^{2}+d^{2}-2 \cdot H 1 \cdot H 2\right)}
\end{gathered}
$$

As in the first case, measuring the phase shift may be ambiguous. The dependence of phase shifts on the zero level from the slant range to the antenna $A_{1}$ is described by the expression:

$$
\Delta \varphi^{(0)}\left(R_{1}\right)=2 \pi \frac{\left(H_{2}^{2}+\left(\left(R_{1}^{2}-H_{1}^{2}\right)^{1 / 2}+d\right)^{2}\right)^{1 / 2}-R_{1}}{\lambda}
$$

Then the value of the phase shift $\Delta \varphi^{(u)}\left(R_{1}\right)$ is used to calculate the difference between the sloping distances is determined from the equation (6). Path $S_{2}$ is used for the calculation $h$ and $r_{1}$ is determined from the expression:

$$
S_{2}=2 R_{1}+\frac{\Delta \varphi^{(u)} \pm 2 \pi m}{2 \pi} \lambda
$$

The value $m$ is chosen by the equation (9).

Still from the spacing antennas, the elements of the resulting images can have some mutual shift. To compensate for this shift is necessary to perform the image correction according to the relation [7]:

$$
J_{R_{1}, x}^{(\kappa o p)}=J_{\frac{R_{1}+R_{2}}{2}, x}^{(2)},
$$

where $R_{2}$ is computed as in equation (10).

Figure 4 shows an example of the recovery bump maps for the considered case when the following system parameters $H_{1}=H_{2}=10 \mathrm{~m}, d=5 \mathrm{~m}$ the maximum altitude of the relief $5 \mathrm{~m}$.

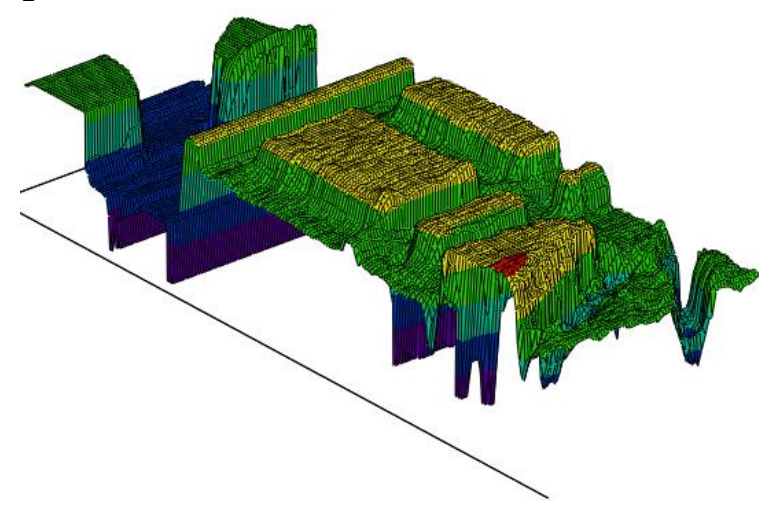

Figure 4. Synthesized 3D image of the surface.

\section{Conclusions}

Solution of a problem of restoration of a landscape`s in a synthetic aperture radar at various configuration of a reception-transmitting path is considered in this work. An original phase unwrapping algorithm based on joint minimization of the estimating error of object's height and 
sloped range in neighboring pixels of the image is proposed. Examples of the restored 3D images are presented.

\section{References}

[1] Richard B, Philipp H 1998 Synthetic aperture radar interferometry Inverse Problems 14(4) R1R54

[2] Fedotov N, Syemov A and Moiseev A 2016 Analysis of conditions that influence the properties of the consructed 3d-image Computer Optics 40(6) 887-894 DOI: 10.18287/2412-6179-201640-6-887-894

[3] Kobernichenko V and Sosnovsky A 2012 Interferometer data processing space radar imagery of high resolution Physics of Wave Processes and Radio Engineering Systems 15(3) 75-83

[4] Vidal-Pantaleoni A, Rafael O and Miguel F 1999 A Comparison of Phase Unwrapping Techniques in Synthetic Aperture Radar Interferometry IEEE International Geoscience and Remote Sensing Symposium 1354-1356

[5] Osmanoglu B and Dixon T 2011 On the importance of path for phase unwrapping in synthetic aperture radar interferometry Applied Optics 50(19) 3205-3220

[6] Richards M 2007 A Beginner's Guide to Interferometric SAR Concepts and Signal Processing IEEE $A$ \& E Systems Magazine 22(9) 5-29

[7] Sosnovsky A and Kobernichenko V 2012 Phase unwrapping algorithms investigation in digital elevation maps generation using space-based InSAR Izvestiya of the Higher Educational Establishments of Russia. Radio electronics 7 84-92

\section{Acknowledgments}

The work is executed at financial support of the Ministry of Education and Science of the Russian Federation, project No. 2.2226.2017/Project Part and project No. 2.9140.2017/Basic Part. The work is performed under financial support of Russian Found of Basic Research, research project No. 15-0799514. 